Research Article

\title{
Diagnostic Performance of a Novel Noninvasive Workup in the Setting of Dry Eye Disease
}

\author{
Luca Vigo, ${ }^{1}$ Marco Pellegrini, ${ }^{2}$ Federico Bernabei, ${ }^{2}$ Francesco Carones, ${ }^{1}$ \\ Vincenzo Scorcia $\mathbb{D}^{3},{ }^{3}$ and Giuseppe Giannaccare $\mathbb{D}^{3}$ \\ ${ }^{1}$ Carones Ophthalmology Center, Milan 20122, Italy \\ ${ }^{2}$ Ophthalmology Unit, S.Orsola-Malpighi University Hospital, University of Bologna, Bologna 40138, Italy \\ ${ }^{3}$ Department of Ophthalmology, University Magna Grocia of Catanzaro, Catanzaro 88100, Italy
}

Correspondence should be addressed to Giuseppe Giannaccare; giuseppe.giannaccare@gmail.com

Received 2 August 2020; Revised 15 November 2020; Accepted 1 December 2020; Published 11 December 2020

Academic Editor: Antonio Benito

Copyright (c) 2020 Luca Vigo et al. This is an open access article distributed under the Creative Commons Attribution License, which permits unrestricted use, distribution, and reproduction in any medium, provided the original work is properly cited.

Purpose. To evaluate the diagnostic performance of a novel noninvasive automated workup employed for the diagnosis of dry eye disease (DED). Methods. One hundred patients with mild to moderate DED and 100 matched control subjects were enrolled in this cross-sectional study. Ocular surface examinations were carried out by means of IDRA Plus (SBM Sistemi, Turin, Italy), which allows the automated evaluation of noninvasive breakup time (NIBUT), lipid layer thickness (LLT), tear meniscus height (TMH), infrared meibography for the measurement of meibomian gland loss (MGL), and blinking analysis. Continuous variables were compared between patients with DED and controls by using the Mann-Whitney $U$ test. The area under the curve (AUC) of receiver operating characteristic curves was calculated. The correlations between ocular surface parameters were evaluated with Pearson correlation analysis. Results. Patients with DED showed significantly lower values of NIBUT, LLT, and TMH compared to controls $(6.9 \pm 2.5$ vs $10.4 \pm 2.4 \mathrm{~s}, P<0.001 ; 64.6 \pm 20.3$ vs $73.4 \pm 21.9 \mathrm{~nm}, P=0.003 ; 0.231 \pm 0.115$ vs $0.289 \pm 0.164, P=0.012$, respectively). Conversely, no significant differences were observed for MGL and blinking analysis (both $P>0.05$ ). NIBUT had the highest diagnostic power $(\mathrm{AUC}=0.841$, sensitivity $=0.89$, and specificity $=0.69)$, followed by LLT $(\mathrm{AUC}=0.621$, sensitivity $=0.89$, and specificity $=0.55)$, TMH $\quad(\mathrm{AUC}=0.606$, sensitivity $=0.57$, and specificity $=0.63)$, blink analysis $(\mathrm{AUC}=0.533$, sensitivity $=0.48$, and specificity $=0.59)$, and MGL $(A U C=0.531$, sensitivity $=0.54$, and specificity $=0.48)$. In patients with DED, NIBUT showed a significant correlation with TMH $(R=0.347, P=0.002)$ and blinking analysis $(R=0.356, P<0.001)$, while blinking analysis was negatively correlated with MGL $(R=-0.315, P=0.008)$. Conclusions. The automated noninvasive workup validated in this study may be a useful tool for reaching a noninvasive diagnosis of DED with a good performance, especially for NIBUT.

\section{Introduction}

Dry eye disease (DED) is a multifactorial disease of tears and ocular surface that represents one of the most frequent ophthalmological complaints, affecting hundreds of millions of people worldwide [1]. Based on the definition by Tear Film and Ocular Surface Society Dry Eye Workshop (TFOS DEWS) II, multiple factors including tear film instability, tear hyperosmolarity, inflammation, and neurosensory abnormalities play a key role in the pathogenesis of DED [2].
No single gold-standard diagnostic marker has yet been established, mainly due to different aspects of the disease including the multifactorial and complex pathogenesis, the poor correlation between symptoms and signs, and the significant fluctuation over time and season of currently available metrics [3-6]. As such, nowadays the diagnosis of DED is reached if ocular discomfort symptoms are present in association with at least one marker of disrupted homeostasis of the ocular surface among corneal staining, tear film instability, and increased tear osmolarity [7]. 
Recently, novel metrics, including among others tear film interferometry, infrared meibography, tear meniscus height, and evaluation of blinking characteristics [8-14], have been developed to complement the diagnosis of DED traditionally reached with slit-lamp examination and vital dye staining. The advantages of these tests include the noninvasive nature and the automated calculation of the results that can provide reliable biomarkers of the disease, avoiding observer bias [15]. However, the current lack of validated cutoff values for reaching the diagnosis of DED hampered their wide adoption in the clinical practice.

The purpose of this study was to evaluate the performance of a novel noninvasive automated workup for reaching the diagnosis of DED.

\section{Materials and Methods}

2.1. Study Design. This cross-sectional study was conducted at the Department of Ophthalmology of the University Magna Græcia of Catanzaro (Italy) between December 2019 and February 2020. The study was performed in accordance with the principles of the Declaration of Helsinki and was approved by the local ethics committee (Comitato Regione Calabria Sezione Area Centro-Protocol n. 280/2019). Consecutive patients over 18 years of age with a confirmed diagnosis of DED who attended the ocular surface office for control visits were screened for enrolment. The diagnosis of DED was reached according to TFOS DEWS II criteria, which require an ocular surface disease index score $\geq 13$ plus one between tear breakup time $($ TBUT) $<10$ seconds or $>5$ spots of corneal staining [7]. Healthy subjects attending our center for routine ophthalmic visits, who were matched by age and gender, were selected as the control group. Exclusion criteria for both groups were contact lens wearing, previous corneal surgery, and active ocular diseases including allergy as well as uncontrolled systemic diseases.

2.2. Ocular Surface Examination. All ocular surface examinations were performed using the newly developed IDRA Plus (SBM Sistemi, Turin, Italy), an all-in-one device which allows the automated measurement of (i) noninvasive breakup time (NIBUT) (Figures 1(a) and 1(b)); (ii) lipid layer thickness (Figures 1(c) and 1(d)); (iii) tear meniscus height (Figures 1(e) and 1(f)); (iv) infrared meibography (Figures $1(\mathrm{~g})$ and $1(\mathrm{~h})$ ); and (v) blinking analysis (Figures 1(i) and 1(j)). In detail, NIBUT was measured without the need for fluorescein dye after asking the patient to blink 3 consecutive times and then hold the eyes open. The measurement was repeated 3 times, and the mean value was recorded. Lipid layer thickness was estimated by observing the interference pattern and colours of the moving lipid tear film. Tear meniscus height was measured along the lower lid margin immediately below the pupil. Infrared meibography was performed after everting the superior eyelid, and meibomian gland loss was defined as the percentage of gland loss in relation to the total tarsal area of the lid. The blinking analysis was performed by recording a 30-second video while the patient was asked to blink naturally by avoiding forced blinking, and the percentage closure of maximal palpebral fissure opening was noted. The tests were performed in the following chronology in order to avoid/ minimize potential confounding effects on the readings of subsequent measurements [16]: blink analysis, tear meniscus height, lipid layer thickness, NIBUT, and infrared meibography.

2.3. Statistical Analysis. The statistical analysis was conducted with R (version 4.0.0) and RStudio (version 1.2.5042) software. Examinations were performed in both eyes of patients, and values from the worst eye according to TFOS DEWS II criteria were used for the statistical analysis. Continuous variables were compared between patients with DED and controls by using the Mann-Whitney $U$ test. Receiver operating characteristic (ROC) curves were drawn to assess the diagnostic significance of ocular surface parameters by using the pROC package [17]. The accuracy of each ocular surface parameter for discriminating patients with DED from controls was evaluated by calculating the area under the curve (AUC). The optimal cutoff value of each parameter was determined as the point on the ROC curve that was nearest to the coordinate $(1,1)$. The correlations between ocular surface parameters were evaluated with Pearson correlation analysis. A Bonferroni correction for multiple comparisons was applied. A $P$ value $<0.05$ was considered statistically significant.

\section{Results}

Overall, 100 eyes of DED patients and 100 eyes of control subjects were included. No significant differences between the two groups were observed for gender distribution $(74 \%$ females in the DED group vs $70 \%$ females in the control group, $P=0.637)$ and age $(50.5 \pm 31.1$ years vs $54.0 \pm 14.7$, $P=0.075)$.

The results of the ocular surface examination in the two groups are reported in Table 1 . Compared to control subjects, patients with DED showed a significantly lower NIBUT $(P<0.001)$, lipid layer thickness $(P=0.003)$, and tear meniscus height $(P=0.012)$. Conversely, no significant differences in meibomian gland loss and blinking analysis were observed (both $P>0.05$ ).

The AUC of ROC curves along with optimal cutoff values with corresponding sensitivity and specificity of the ocular surface parameters analyzed is reported in Table 2: NIBUT had the highest diagnostic power $(\mathrm{AUC}=0.841$, sensitivity $=0.89$, and specificity $=0.69)$, followed by lipid layer thickness $(\mathrm{AUC}=0.621$, sensitivity $=0.89$, and specificity $=0.55)$, tear meniscus height $(\mathrm{AUC}=0.606$, sensitivity $=0.57$, and specificity $=0.63)$, blinking analysis $(\mathrm{AUC}=0.533$, sensitivity $=0.48$, and specificity $=0.59)$, and meibomian gland loss $(\mathrm{AUC}=0.531$, sensitivity $=0.54$, and specificity $=0.48)$. Figure 2 shows the ROC curves of NIBUT, lipid layer thickness, and tear meniscus height.

In patients with DED, NIBUT showed a significant correlation with tear meniscus height $(R=0.347, P=0.002)$ and blinking analysis $(R=0.356, P<0.001)$; moreover, 


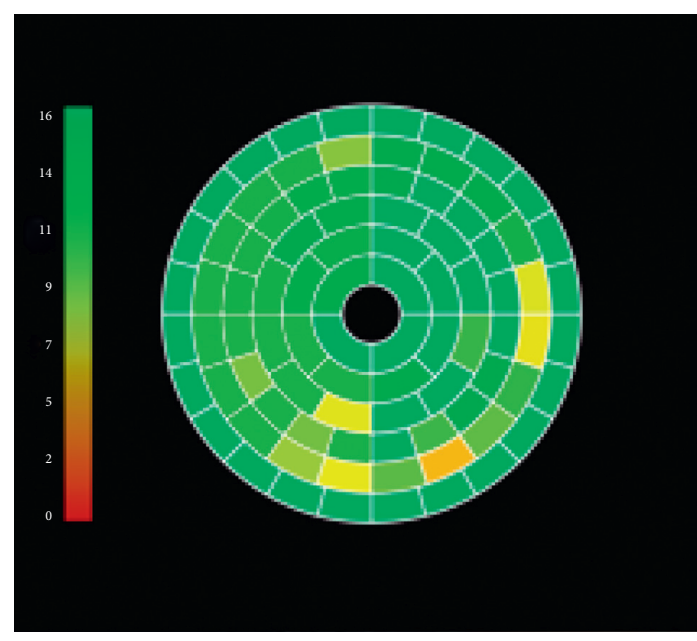

(a)

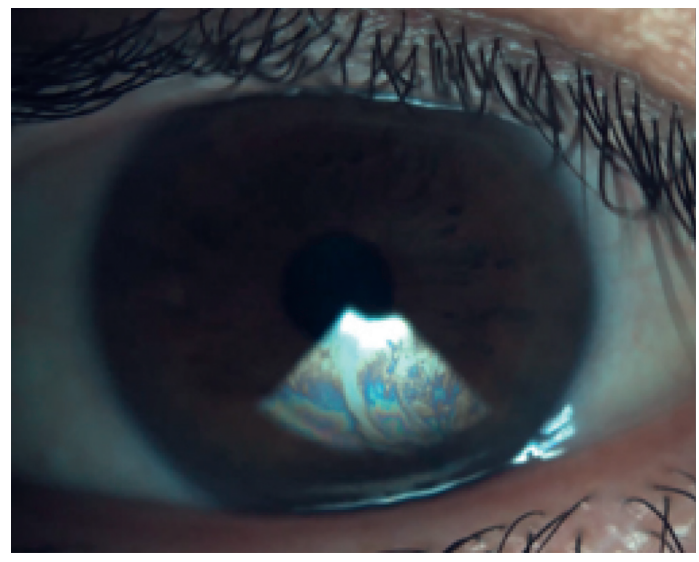

(c)

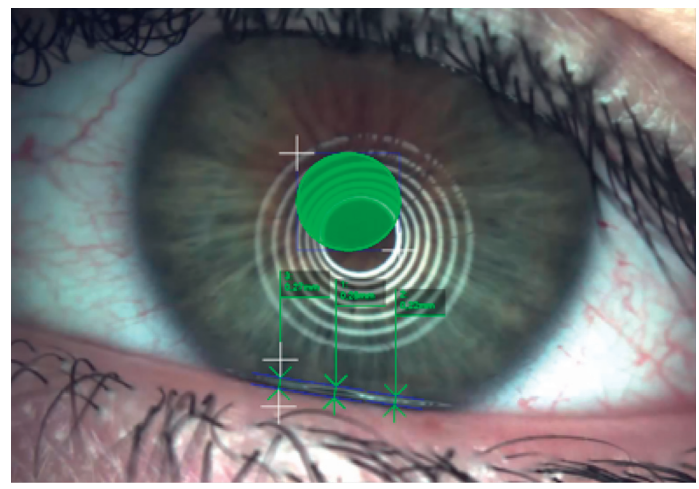

(e)

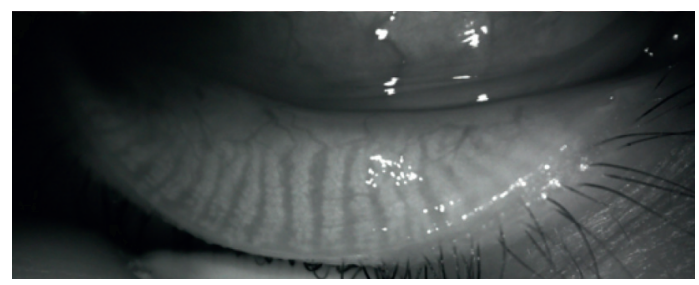

(g)

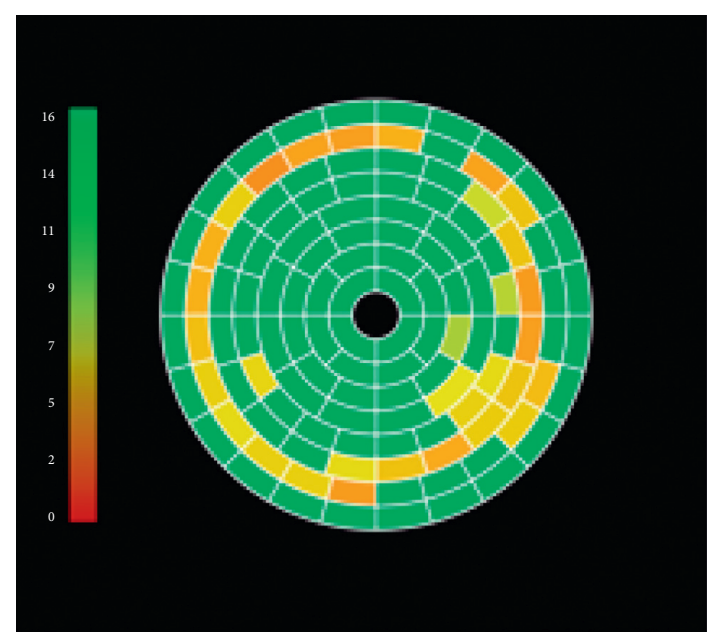

(b)

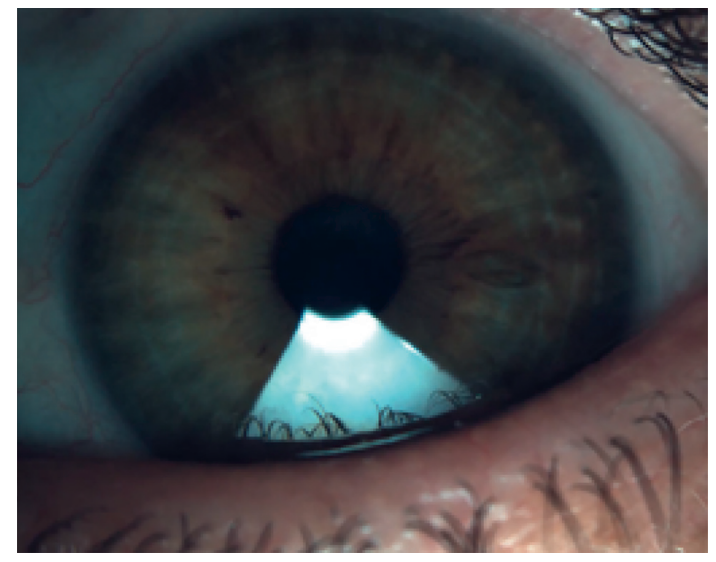

(d)

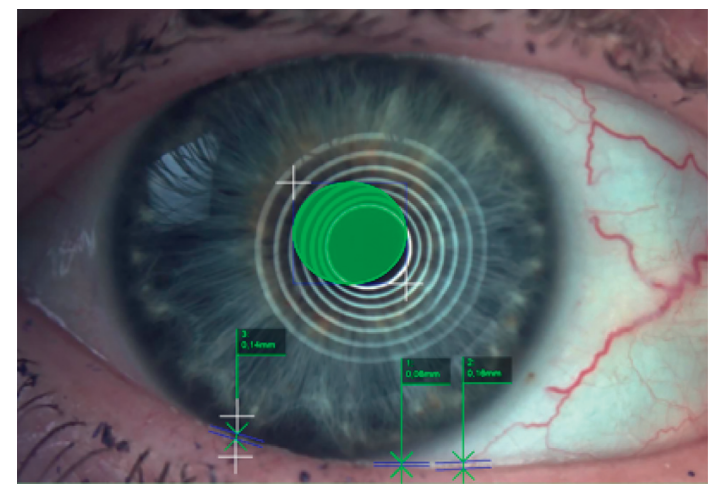

(f)

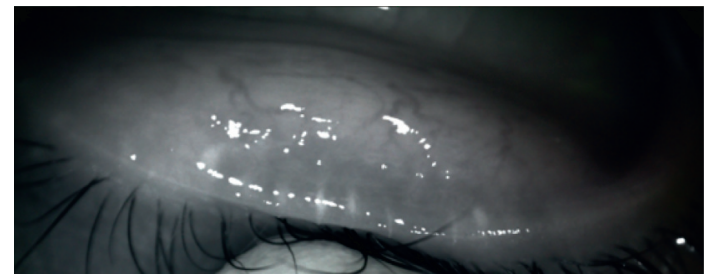

(h)

Figure 1: Continued. 


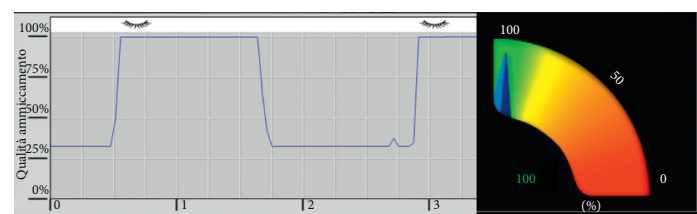

(i)

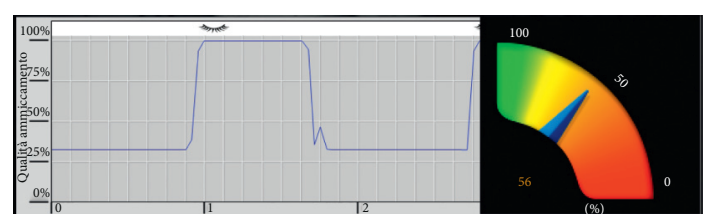

(j)

FiguRe 1: Ocular surface workup in a representative control subject (a, c, e, g, i) and in a patient with dry eye disease (b, d, f, h, j). (a, b) Measurement of noninvasive breakup time. (c, d) Tear film interferometry for the measurement of lipid layer thickness. (e, f) Measurement of tear meniscus height. (g, h) Infrared meibography. (i, j) Blink analysis.

TABLE 1: Ocular surface parameters in patients with dry eye disease and control subjects.

\begin{tabular}{lccc}
\hline Parameter & Dry eye group $(n=100)$ & Control group $(n=100)$ & $P$ value \\
\hline NIBUT $(\mathrm{s})$ & $6.9 \pm 2.5$ & $10.4 \pm 2.4$ & $\mathbf{2}$ \\
Lipid layer thickness $(\mathrm{nm})$ & $64.6 \pm 20.3$ & 0.001 \\
Tear meniscus height $(\mathrm{mm})$ & $0.231 \pm 0.115$ & $0.289 \pm 0.164$ & $\mathbf{0 . 0 0 3}$ \\
Meibomian gland loss $(\%)$ & $22.4 \pm 12.9$ & $20.3 \pm 11.4$ & $\mathbf{0 . 0 1 2}$ \\
Blink analysis & $85.0 \pm 19.5$ & $87.2 \pm 18.8$ & 0.458 \\
\hline
\end{tabular}

TABLE 2: Area under the curve (AUC) with 95\% confidence intervals (CIs), optimal cutoff values, and corresponding sensitivity and specificity for the analyzed ocular surface parameters.

\begin{tabular}{|c|c|c|c|c|c|}
\hline Parameter & AUC & $95 \% \mathrm{CI}$ & Cutoff & Sensitivity & Specificity \\
\hline NIBUT (s) & 0.841 & $0.786-0.895$ & 7.75 & 0.89 & 0.69 \\
\hline Lipid layer thickness (nm) & 0.621 & $0.543-0.699$ & 66.5 & 0.64 & 0.55 \\
\hline Tear meniscus height $(\mathrm{mm})$ & 0.606 & $0.527-0.685$ & 0.225 & 0.57 & 0.63 \\
\hline Meibomian gland loss (\%) & 0.531 & $0.450-0.611$ & 17.5 & 0.54 & 0.48 \\
\hline Blink analysis & 0.533 & $0.460-0.606$ & 99.0 & 0.48 & 0.59 \\
\hline
\end{tabular}

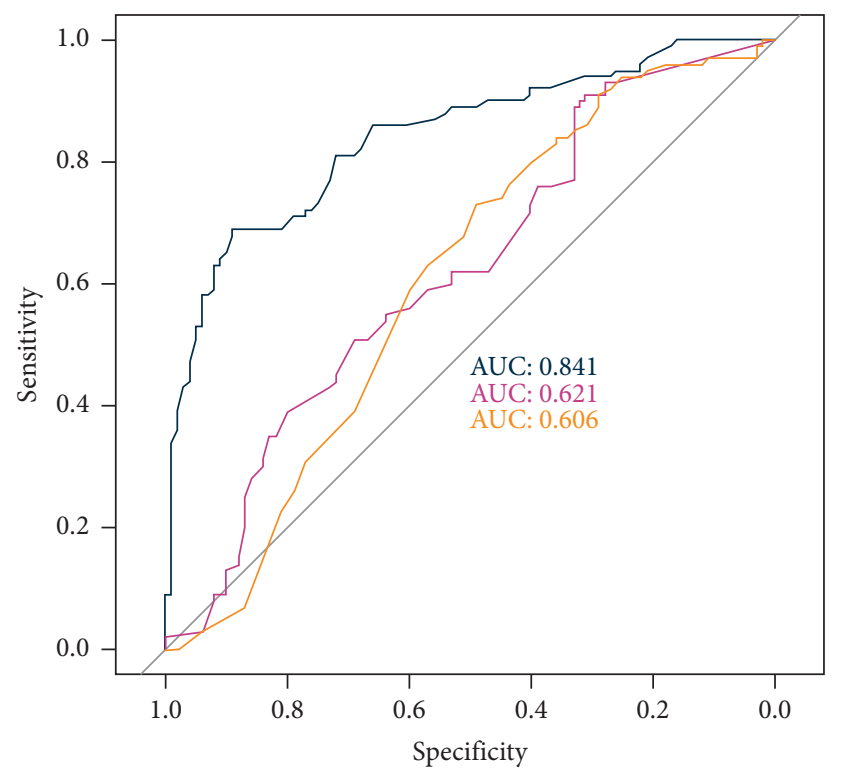

- NIBUT

— Lipid layer thickness

_ Tear meniscus height

FIGURE 2: Receiver operating characteristic curves with area under the curve of noninvasive breakup time, lipid layer thickness, and tear meniscus height for the diagnosis of dry eye disease. blinking analysis was negatively correlated with meibomian gland loss $(R=-0.315, P=0.008)$. No other significant correlations were observed.

\section{Discussion}

The prevalence of DED varies consistently across different population, and this is partially due to the heterogeneity of diagnostic criteria used in different studies [1]. To address this issue, the TFOS DEWS II guidelines developed a consensus diagnostic battery of tests for DED including breakup time, tear osmolarity, ocular surface staining, and symptomatology [7]. Nevertheless, the DEWS II Diagnostic Methodology Subcommittee acknowledged the lack of a gold-standard test to diagnose DED and the need of identifying new reliable biomarkers [7]. In the same report, it has been highlighted that studies evaluating novel diagnostic tests are frequently affected by selection and spectrum biases. The former occurs when a novel test is compared to established ones that were used as inclusion criteria, resulting in apparently poor performance. The latter refers to the exclusion from clinical trials of patients with mild disease, with overestimation of the diagnostic performance. Conversely, to avoid both these biases and obtain reliable estimates of the diagnostic performance, novel tests should be developed and validated using data from the population 
in which they are intended to be used [18]. Therefore, in the present study, we included consecutive patients with a confirmed diagnosis of DED presenting to our center for routine control visits. Since DED diagnosis had been already reached previously, we did not use conventional tests to select and grade patients. This resulted in the inclusion of a broad population of mild to moderate DED patients, producing results that are generalizable to real-life clinical practice.

Patients with DED showed significantly lower values of NIBUT, lipid layer thickness, and tear meniscus height compared to controls, while no differences in meibomian gland loss and blinking analysis were observed. The ROC analysis showed that NIBUT was the parameter with the highest sensitivity and specificity to diagnose DED. Lipid layer thickness and tear meniscus height had moderate diagnostic utility, while the performances of meibomian gland loss and blinking analysis were poor.

In agreement with these results, also previous works focused on both hyposecretory [19] and evaporative DED [10] found that NIBUT was the best single diagnostic test for reaching the diagnosis. Our results further confirm the role of tear film instability measurement as a reliable indicator of DED diagnosis. Compared to conventional breakup time, NIBUT has the advantage of avoiding contact with the ocular surface as well as disruption of the tear film induced by fluorescein instillation [20]. Interestingly, the optimal NIBUT cutoff value in this study was 7.75 seconds, which is lower than the cutoff of 10 seconds proposed in previous works $[7,21]$.

Tear meniscus height and lipid layer thickness showed moderate diagnostic performance to differentiate DED from controls. Singh and colleagues recently reported higher accuracy of tear meniscus height (sensitivity 0.98 and specificity 0.96) in patients with moderate to severe DED [13]. Not surprisingly, the performance of this parameter in patients with milder diseases, like those included in our study, was found to be lower. Conversely, the previously reported accuracy of lipid layer thickness for the diagnosis of meibomian gland dysfunction (sensitivity 0.65 and specificity 0.63 ) is consistent with the results of our study [22].

No differences in meibography between DED and controls were observed in this study. Although we did not classify patients according to the subtype of DED (aqueous, evaporative, or mixed), this finding could be explained by the limited number of patients with evaporative DED included in the study. In fact, it has been shown that meibomian gland changes are usually more pronounced in meibomian gland dysfunction compared to DED of other types [23].

Although blinking analysis showed limited diagnostic utility, this parameter showed a significant but relatively weak correlation with both NIBUT and meibomian gland loss. Jie and coauthors reported similar associations and speculated that incomplete blinking could lead to inadequate meibomian gland expression and subsequent tear film instability [14]. It should be noted that a standardized methodology to evaluate incomplete blinking has not yet been developed. We measured the percentage of eye closure while patients blinking spontaneously, but also other methods such as the incomplete blink rate might provide additional information for the characterization of eyelid dynamics $[24,25]$.

This study suffers from some limitations that deserve mentioning. In particular, in order to best reflect everyday practice, we included patients with a confirmed diagnosis of DED regardless of disease severity and/or DED subtype. Further studies with more rigorous inclusion criteria are needed to evaluate the possible changes of diagnostic performance in different DED scenarios. Furthermore, future studies are warranted to investigate the correlation between the results obtained with this new noninvasive diagnostic device and DED clinical and molecular findings.

\section{Conclusions}

The automated noninvasive workup presented and validated in this study may be a useful tool to diagnose DED with good values of sensitivity and specificity, especially for NIBUT. Furthermore, since the effects of this workup on volume or properties of the tear film are negligible, it can be used as an effective screening tool for discriminating healthy subjects from patients affected or at risk for DED, before proceeding with invasive ocular surface examinations required for a better characterization of the disease.

\section{Data Availability}

The data that support the findings of this study are available from the corresponding author upon reasonable request.

\section{Conflicts of Interest}

The authors declare that they have no conflicts of interest.

\section{Authors' Contributions}

Luca Vigo and Marco Pellegrini equally contributed to this work and share primary authorship.

\section{References}

[1] F. Stapleton, M. Alves, V. Y. Bunya et al., "TFOS DEWS II epidemiology report," The Ocular Surface, vol. 15, no. 3, pp. 334-365, 2017.

[2] J. P. Craig, K. K. Nichols, E. K. Akpek et al., "TFOS DEWS II definition and classification report," The Ocular Surface, vol. 15, no. 3, pp. 276-283, 2017.

[3] J. E. Moore, J. E. Graham, E. A. Goodall et al., "Concordance between common dry eye diagnostic tests," British Journal of Ophthalmology, vol. 93, p. 66, 2009.

[4] G. Fuentes-Paez, J. M. Herreras, Y. Cordero, A. Almaraz, M. J. Gonzalez, and M. Calonge, "Lack of concordance between dry eye syndrome questionnaires and diagnostic tests," Archivos de la Sociedad Espanola de Oftalmologia, vol. 86, p. 37, 2011.

[5] B. D. Sullivan, L. A. Crews, E. M. Messmer et al., "Correlations between commonly used objective signs and symptoms for the diagnosis of dry eye disease: clinical implications," Acta Ophthalmologica, vol. 92, p. 16, 2014. 
[6] P. Aragona, G. Giannaccare, R. Mencucci, P. Rubino, E. Cantera, and M. Rolando, "Modern approach to the treatment of dry eye, a complex multifactorial disease: a P.I.C.A.S.S.O. board review," British Journal of Ophthalmology, vol. 23, 2020.

[7] J. S. Wolffsohn, R. Arita, R. Chalmers et al., “TFOS DEWS II diagnostic methodology report," The Ocular Surface, vol. 15, no. 3, pp. 539-574, 2017.

[8] J. J. Nichols, K. K. Nichols, B. Puent, M. Saracino, and G. L. Mitchell, "Evaluation of tear film interference patterns and measures of tear break-up time," Optometry and Vision Science, vol. 79, no. 6, pp. 363-369, 2002.

[9] Y. Ban, S. Shimazaki-Den, K. Tsubota, and J. Shimazaki, "Morphological evaluation of meibomian glands using noncontact infrared meibography," The Ocular Surface, vol. 11, no. 1, pp. 47-53, 2013.

[10] G. Giannaccare, L. Vigo, M. Pellegrini, S. Sebastiani, and F. Carones, "Ocular surface workup with automated noninvasive measurements for the diagnosis of meibomian gland dysfunction," Cornea, vol. 37, no. 6, pp. 740-745, 2018.

[11] G. Giannaccare, F. Bonifazi, S. Sebastiani et al., "Meibomian gland dropout in hematological patients before hematopoietic stem cell transplantation," Cornea, vol. 37, no. 10, pp. 1264-1269, 2018.

[12] L. Vigo, L. Taroni, F. Bernabei et al., "Ocular surface workup in patients with meibomian gland dysfunction treated with intense regulated pulsed light," Diagnostics, vol. 9, no. 4, p. 147, 2019.

[13] A. Singh, M. Vanathi, A. Kishore, N. Gupta, and R. Tandon, "Evaluation of strip meniscometry, tear meniscus height and depth in the diagnosis of dry eye disease in Asian Indian eyes," The Ocular Surface, vol. 17, no. 4, pp. 747-752, 2019.

[14] Y. Jie, R. Sella, J. Feng, M. L. Gomez, and N. A. Afshari, "Evaluation of incomplete blinking as a measurement of dry eye disease," The Ocular Surface, vol. 17, no. 3, pp. 440-446, 2019.

[15] N. S. Roy, Y. Wei, E. Kuklinski, and P. A. Asbell, “The growing need for validated biomarkers and endpoints for dry eye clinical research," Investigative Opthalmology \& Visual Science, vol. 58, no. 6, pp. BIO1-BIO19, 2017.

[16] S. Koh, C. Ikeda, S. Watanabe et al., "Effect of non-invasive tear stability assessment on tear meniscus height," Acta Ophthalmologica, vol. 93, pp. 135-139, 2015.

[17] X. Robin, N. Turck, A. Hainard et al., "pROC: an open-source package for R and S+ to analyze and compare ROC curves," BMC Bioinformatics, vol. 12, p. 77, 2011.

[18] J. A. Usher-Smith, S. J. Sharp, and S. J. Griffin, "The spectrum effect in tests for risk prediction, screening, and diagnosis," BMJ, vol. 353, 2016.

[19] H. Pult, C. Purslow, and P. J. Murphy, "The relationship between clinical signs and dry eye symptoms," Eye, vol. 25, no. 4, pp. 502-510, 2011.

[20] L. E. Downie, "Automated tear film surface quality breakup time as a novel clinical marker for tear hyperosmolarity in dry eye disease," Investigative Ophthalmology \& Visual Science, vol. 56, p. 7260e8, 2015.

[21] L. S. Mengher, A. J. Bron, S. R. Tonge, and D. J. Gilbert, “A non-invasive instrument for clinical assessment of the precorneal tear film stability," Current Eye Research, vol. 4, 1985.

[22] D. Finis, N. Pischel, S. Schrader, and G. Geerling, "Evaluation of lipid layer thickness measurement of the tear film as a diagnostic tool for Meibomian gland dysfunction," Cornea, vol. 32, no. 12, pp. 1549-1553, 2013.
[23] R. Arita, K. Itoh, S. Maeda, K. Maeda, A. Tomidokoro, and S. Amano, "Efficacy of diagnostic criteria for the differential diagnosis between obstructive meibomian gland dysfunction and aqueous deficiency dry eye," Japanese Journal of Ophthalmology, vol. 54, 2010.

[24] T. Wan, X. Jin, L. Lin, Y. Xu, and Y. Zhao, "Incomplete blinking may attribute to the development of meibomian gland dysfunction," Current Eye Research, vol. 41, no. 2, pp. 179-185, 2016.

[25] J. D. Rodriguez, K. J. Lane, G. W. Ousler, E. Angjeli, L. M. Smith, and M. B. Abelson, "Blink: characteristics, controls, and relation to dry eyes," Current Eye Research, vol. 43, no. 1, pp. 52-66, 2018. 